\title{
Sequential irinotecan hydrochloride/S-1 for S-1-resistant inoperable gastric cancer: A feasibility study
}

\author{
TAKENORI TAKAHATA ${ }^{1}$, JUGOH ITOH $^{1}$, TAROH SATOH $^{5}$, ATSUSHI ISHIGURO ${ }^{1}$, \\ YOSHIFUMI MATSUMOTO ${ }^{1}$, SATOSHI TANAKA ${ }^{1}$, SOH SAITOH $^{3}$, HIROSHI TOHNO ${ }^{4}$, \\ SHINSAKU FUKUDA ${ }^{2}$, YASUO SAIJO ${ }^{1}$ and YUH SAKATA ${ }^{6}$ \\ Departments of ${ }^{1}$ Medical Oncology, and ${ }^{2}$ Gastroenterology and Hematology, Hirosaki University \\ Graduate School of Medicine, 5 Zaifu-cho, Hirosaki 036-8562; ${ }^{3}$ Department of Gastroenterology and Medical Oncology, \\ Aomori Prefectural Hospital, 2-1-1 Higashitsukurimichi, Aomori 030-8553; \\ ${ }^{4}$ Department of Internal Medicine, Hirosaki City Hospital, 3-8-1 Omachi, Hirosaki 036-8004; \\ ${ }^{5}$ Department of Medical Oncology, Kinki University School of Medicine, 377-2 Ohnohigashi, Osakasayama 589-8511; \\ ${ }^{6}$ Department of Medical Oncology, Misawa City Hospital, 164-65 Horiguchi, Misawa 033-0022, Japan
}

Received May 26, 2011; Accepted September 28, 2011

DOI: $10.3892 / \mathrm{ol} .2011 .435$

\begin{abstract}
Irinotecan hydrochloride (CPT-11) is reported to be involved in the downregulation of thymidylate synthase (TS), a target molecule of 5-fluorouracil (5-FU) and oral fluoropyrimidine S-1. Therefore, we hypothesized that a preceding administration of CPT-11 against S-1-resistant tumors may recover sensitivity to $S-1$. To this end, we planned a S-1/CPT-11 sequential therapy as a feasibility study in S-1-refractory gastric cancer patients. In the first course, CPT-11 was administered intravenously at $150 \mathrm{mg} / \mathrm{m}^{2}$ on days 1 and 15 . Subsequently, S-1 was administered orally for 4 weeks from day 29 to 57 , followed by a 2-week interval (sequential S-1/CPT-11). When the tumor showed a complete response (CR) or partial response (PR), the same dose of S-1 monotherapy was continued unless progressive disease (PD) was observed. When the response was stable disease (SD), S-1 was administered at the same dose for just 2 weeks (days 1-15), no drug was administered for the following 2 weeks (4-week cycle) and CPT-11 was administered intravenously at $100 \mathrm{mg} / \mathrm{m}^{2}$ on days 1 and 15 (concurrent S-1/ CPT-11) unless PD was observed. In the case of PD, the study was terminated. The primary endpoint was an antitumor effect and secondary endpoints were median survival time (MST), progression-free survival (PFS), time-to-treatment failure (TTF) and safety. The response rate (RR) following the first course was only $5.9 \%$ and the most positive RR was $11.8 \%$. The MST, median TTF and PFS were 381, 69 and 71 days, respectively. Leukocytopenia was observed in more than half of the patients. Since the RR was lower than estimated in an
\end{abstract}

Correspondence to: Dr Takenori Takahata, Department of Medical Oncology, Hirosaki University Graduate School of Medicine, 5 Zaifu-cho, Hirosaki 036-8562, Japan

E-mail: takatake@cc.hirosaki-u.ac.jp

Key words: CPT-11, S-1, gastric cancer interim analysis, the trial was terminated and the protocol was concluded to be unfeasible.

\section{Introduction}

Gastric cancer treated by surgical resection is radical and shows a favorable prognosis; however, when cases are inoperable due to the advanced stage, the prognosis is poor, with a $10 \%$-year survival rate. Chemotherapies against gastric cancer have been developed as combination chemotherapies since the 1980s. In the 1990s, phase III randomized comparative studies between best supportive care (BSC) and chemotherapies revealed a significant improvement in overall survival rates (1-3).

Irinotecan hydrochloride (CPT-11), synthesized from camptothecin contained in the Chinese tree Camptotheca acuminate, inhibits type I topoisomerase and DNA synthesis, and thus demonstrates antitumor effects. In Japan, CPT-11 has been approved for various types of cancer, including small and non-small cell lung cancer, uterine cervical cancer, ovarian cancer, gastric cancer, colorectal cancer, breast cancer, squamous cell carcinoma of the skin, and malignant lymphoma. The overall response rate (RR) to CPT-11 monotherapy is reported to be $23.3 \%$ in late phase II trials for advanced gastric cancer (4).

S-1 is an oral anticancer drug containing a combination of tegafur (FT), a prodrug of 5-fluorouracil (5-FU), 5-chloro2,4-dihydroxypyrimidine (CDHP) that inhibits the activity of dihydropyrimidine dehydrogenase (DPD), and potassium oxonate (Oxo), which reduces the gastrointestinal toxicity of 5-FU. The S-1 monotherapy for advanced gastric cancer revealed non-inferiority to 5-FU infusion in the JCOG9912 study. A subsequent study revealed that a combination of S-1 and cisplatin (CDDP) is superior to the S-1 monotherapy (5). Since these trials, S-1 plus CDDP has been one of the standard chemotherapies against advanced gastric cancer in Japan.

S-1 or S-1-containing regimens are used in adjuvant chemotherapy following surgery or in first-line chemotherapy 
for inoperable gastric cancer. One of the mechanisms of resistance against S-1 is thought to be an increase of thymidylate synthase (TS) activity, which is a target of 5-FU in tumor cells. A study revealing that irinotecan downregulates intratumoral TS and makes 5-FU more effective in human colon cancer xenografts suggests the possibility of overcoming S-1 resistance by adding irinotecan (6). In this context, we planned a feasibility study in which S-1-pretreated gastric cancer patients were treated with a combination chemotherapy of S-1 and CPT-11 as a feasibility test.

\section{Materials and methods}

Patient selection. Patients with histologically confirmed gastric cancer with measureable or evaluable lesions were eligible for this study. Patients were required to have been previously treated with a first-line chemotherapy containing S-1, but not CPT-11. Other eligibility criteria were: $20-75$ years old, Eastern Cooperative Oncology Group (ECOG) performance status (PS) of 0-2, capable of oral intake, white blood cell count (WBC) of 3,500-12,000/ $\mu 1$, neutrocyte count (Neu) $>2,000 / \mu 1$, platelet count (PLT) $>100,000 / \mu 1$, hemoglobin (Hb) level $>9.0 \mathrm{~g} / \mathrm{dl}$, serum total bilirubin (T-bil) $<1.5 \mathrm{mg} / \mathrm{dl}$, serum aspartate aminotransferase (AST) and serum alanine aminotransferase (ALT) $<2$ times the normal limit, serum creatinine within the normal limit, creatinine clearance calculated with the Cockcroft-Gault equation $>50 \mathrm{ml} / \mathrm{min}$, survival expectancy of at least 3 months, and written informed consent for this study.

Study design. This study is a multicenter, non-randomized, open-label feasibility study. An overview of the study is shown in Fig. 1. In the first course, CPT-11 was administered intravenously at $150 \mathrm{mg} / \mathrm{m}^{2}$ on days 1 and 15 . Subsequently, S-1 was administered orally for 4 weeks from day 29 to 57, followed by a 2-week interval (sequential S-1/CPT-11). The dosage of S-1 was based on body surface area (BSA): $40 \mathrm{mg}$ (BSA $<1.25$ $\mathrm{m}^{2}$ ), $50 \mathrm{mg}\left(\right.$ BSA $\geq 1.25$ and $<1.5 \mathrm{~m}^{2}$ ) or $60 \mathrm{mg}$ b.i.d. (BSA $\geq 1.5 \mathrm{~m}^{2}$ ). A CT scan was performed during this 2-week interval to evaluate the tumor response according to the Response Evaluation Criteria in Solid Tumors (RECIST). When the tumor showed a complete response (CR) or partial response (PR) in the first course, the same dose of S-1 monotherapy was continued unless progressive disease (PD) was observed. When the response was stable disease (SD), S-1 was administered at the same dose for only 2 weeks (days 1-15), no drug was administered for the following 2 weeks (4-week cycle) and CPT-11 was administered intravenously at $100 \mathrm{mg} / \mathrm{m}^{2}$ on days 1 and 15 (concurrent S-1/CPT-11) unless PD was observed. In the case of PD, the study was terminated. There was no restriction in third-line chemotherapy. Adverse events were evaluated using the National Cancer Institute Common Terminology Criteria for Adverse Events (NCI-CTCAE), version 3.0. The protocol was approved by the Institutional Review Board of Hirosaki University School of Medicine and other institutes. The primary endpoint of this study was an antitumor effect and secondary endpoints were median survival time (MST), progression-free survival (PFS), time-to-treatment failure (TTF) and safety.

Statistical analysis. For prognostic values, the MST, PFS and TTF were calculated using the Kaplan-Meier method from

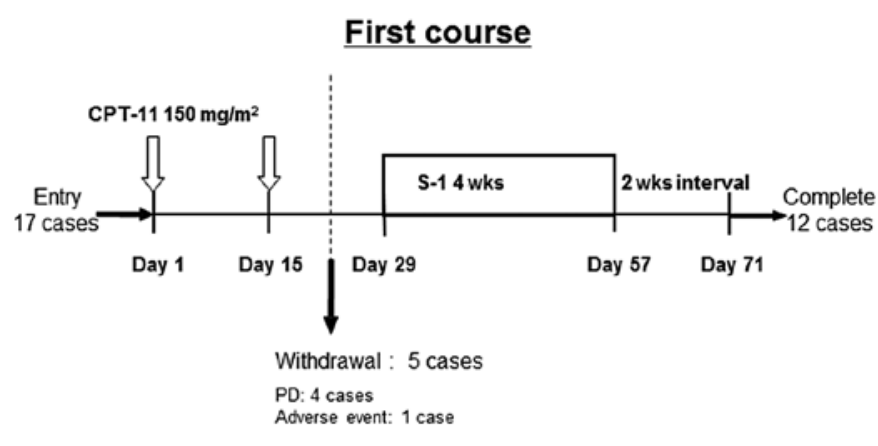

Second course and thereafter

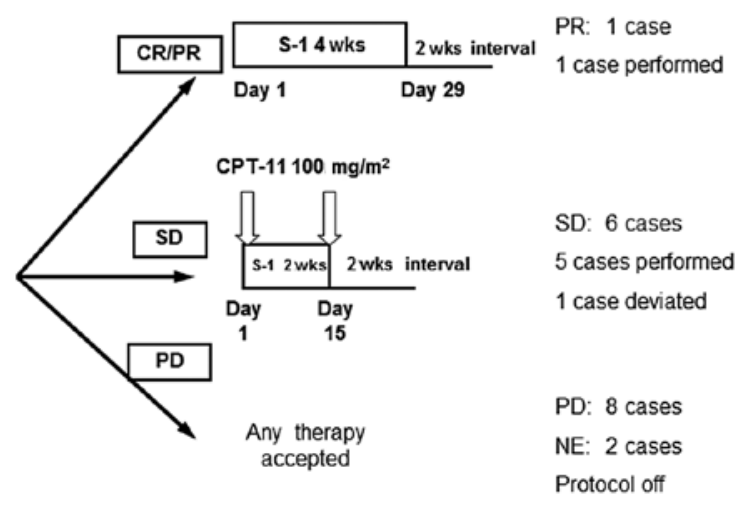

Figure 1. Protocol design of the CPT-11/S-1 sequential study. In the first course, $150 \mathrm{mg} / \mathrm{m}^{2} \mathrm{CPT}-11$ (days 1 and 15) and 4-week S-1 (day 29 to 57) was administered followed by a 2-week interval (sequential S-1/CPT-11). In case of CR or PR, the same dose of S-1 monotherapy was continued unless PD was observed. For SD, S-1 (days 1-15), and $100 \mathrm{mg} / \mathrm{m}^{2}$ CPT-11 (days 1 and 15) was administered in a 4-week cycle (concurrent S-1/CPT-11) unless PD was observed. In the case of PD, the study was terminated.

the date of registration. The estimated RR and threshold RR were set at 15 and 5\%, respectively, and the calculated minimum sample size was estimated to be 43 , with an $\alpha$ value of 0.05 and a $\beta$ value of 0.20 . We estimated that the number of possible patient exclusions or dropouts would be 2 , and the sample size was increased to 45 . In the planned interim analysis, when the number of response cases was $\leq 1$ out of 12 enrolled cases, the study would be terminated since the estimated RR would be $<5 \%$.

\section{Results}

Patient characteristics. Patient characteristics are shown in Table I. A total of 17 patients (14 males and 3 females), were enrolled in this study between November 2004 and June 2006. The median age was 63 years, and a PS of 0/1 was observed in $14 / 3$ patients, respectively. Histological results revealed that 8 patients had intestinal and 9 had diffuse cancer types. Eleven patients had primary lesions and 5 did not, and no information was available in 1 case. Prior to enrollment in this study, 7 patients underwent surgical treatment and 10 patients did not. Previous chemotherapy regimens were S-1 monotherapy $(n=9), S-1 / C D D P(n=7)$ and S-1/taxotere $(T X T)(n=2)$. None of the patients had received radiotherapy. 
Table I. Patient characteristics.

\begin{tabular}{lc}
\hline Characteristics & Patient no. \\
\hline Gender (M/F) & $14 / 3$ \\
Age (mean/median) & $59.6 / 63$ \\
Performance status (0/1) & $14 / 3$ \\
Untreated/recurrence & $10 / 7$ \\
Histology (intestinal/diffuse) & $8 / 9$ \\
Primary lesion (+/-/unknown) & $11 / 5 / 1$ \\
Metastasis & \\
Lung & 2 \\
Liver & 8 \\
Bone & 1 \\
Abdominal lymph node & 11 \\
Other & 5 \\
Radiotherapy (+/-) & $0 / 17$ \\
Surgical operation (+/-) & $7 / 10$ \\
Prior chemotherapy regimen & \\
S-1 & 8 \\
S-1/CDDP & 7 \\
S-1/taxotere & 2 \\
Detail of S-1 resistance & 5 \\
Unresectable and formerly effective & 5 \\
Unresectable and formerly resistant & \\
Recurrence during adjuvant chemotherapy & 5 \\
Recurrence after adjuvant chemotherapy & \\
\hline & \\
\hline
\end{tabular}

Tumor response and survival rate. Five out of 17 patients ceased chemotherapy halfway through the first course of treatment due to PD in 4 patients and an adverse event in 1 patient. Twelve patients completed the first course. However, 5 patients out of 12 dropped out due to PD following completion of the first course. The tumor response following the first cycle is shown in Table II. Only 1 patient showed PR following the first course (RR, 5.9\%; 95\% CI, 0.1-28.7). The PR patient received 2 cycles of S-1 monotherapy and the 5 SD patients received a median of 2 cycles (mean 3.6 cycles; range 1-6) of concurrent $\mathrm{S}-1 / \mathrm{CPT}-11$. One of the $5 \mathrm{SD}$ patients showed PR during the S-1/CPT-11 chemotherapy. Therefore, the most positive tumor response rate was $2 \mathrm{PR}, 5 \mathrm{SD}, 8 \mathrm{PD}$ and 2 not evaluable (NE) (RR, 11.8\%; 95\% CI, 1.5-31.4) (Table II). An overall survival curve is shown in Fig. 2. Regarding the prognostic analysis by the log-rank test, the MST, median TTF and PFS were 381, 69 and 71 days, respectively.

Retrospectively, we performed a subset analysis using stratification by different patient background of S-1 resistance. We compared the S-1-sensitive group (for which the first-line chemotherapy was effective or 6-month adjuvant chemotherapy was completed) and the S-1-resistant group (for which the first-line chemotherapy was not effective or adjuvant chemotherapy was not completed). However, no significant difference in MST, TTF or PFS was observed in the subset analysis.

After 17 cases had enrolled in this study, an interim analysis was performed according to the initial schedule. Since the RR following the first course was only $5.9 \%$ and 10 cases received
Table II. Tumor response.

A, After the first course.

\begin{tabular}{lcccccc}
\hline $\mathrm{n}$ & $\mathrm{CR}$ & $\mathrm{PR}$ & $\mathrm{SD}$ & $\mathrm{PD}$ & $\mathrm{NE}$ & $\mathrm{RR}$ \\
17 & 0 & 1 & 6 & 8 & 2 & $5.9 \%$ \\
\hline $\mathrm{B}$, Overall. & & & & & \\
\hline $\mathrm{n}$ & $\mathrm{CR}$ & $\mathrm{PR}$ & $\mathrm{SD}$ & $\mathrm{PD}$ & $\mathrm{NE}$ & $\mathrm{RR}$ \\
17 & 0 & 2 & 5 & 8 & 2 & $11.8 \%$ \\
\hline
\end{tabular}

CR, complete response; PR, partial response; $\mathrm{SD}$, stable disease; $\mathrm{PD}$, progressive disease; NE, not evaluable; RR, response rate.

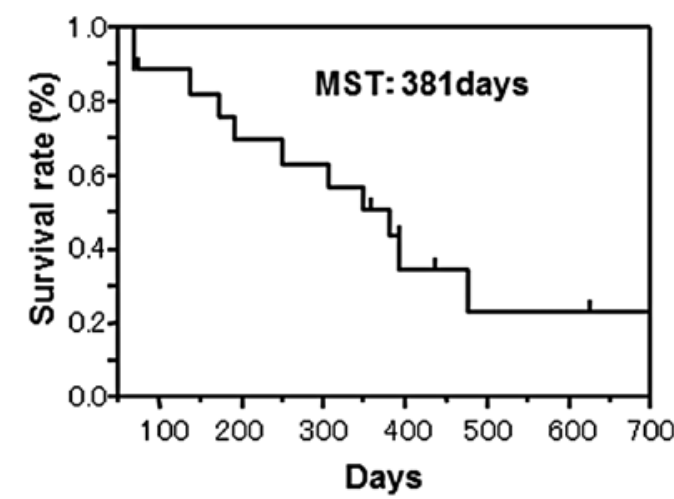

Figure 2. Survival curve of the CPT-11/S-1 sequential study. The MST was calculated using the Kaplan-Meier method from the date of registration and was 381 days.

only the first course of chemotherapy, this protocol was evaluated as being unfeasible.

Toxicity. Observed toxicities associated with this chemotherapy protocol are shown in Table III. The most common toxicities were leukocytopenia and neutropenia in both the first and subsequent courses (58.8 and 66.7\%, respectively). Nausea and vomiting were also common. One patient had G4 neutropenia and a further patient had G4 anorexia. Diarrhea, a common adverse event of CPT-11 and S-1, was observed more frequently in concurrent S-1/CPT-11 chemotherapy, but not in the sequential regimen. Chemotherapy-related mortality was not observed.

\section{Discussion}

This trial tested the feasibility of sequential CPT-11 and S-1 in S-1-refractory gastric cancer patients. The RR following the first course was only $5.9 \%$ and the most positive RR was $11.8 \%$ (Table II). The MST was 381 days (Fig. 2) and the median TTF and PFS were 69 and 71 days, respectively. Therapyrelated mortality was not observed, although leukocytopenia and neutropenia were observed in over half of the patients (Table III), indicating moderate toxicity of this protocol. Since the RR following the first course was lower than that estimated in an interim analysis, the trial was terminated. 
Table III. Adverse events during the course of the study.

A, First course.

\begin{tabular}{|c|c|c|c|c|c|c|}
\hline $\mathrm{n}=17$ & G1 & $\mathrm{G} 2$ & G3 & G4 & All grades (\%) & $>\mathrm{G} 3(\%)$ \\
\hline Leukocytopenia & 3 & 5 & 2 & 0 & 58.8 & 11.8 \\
\hline Neutropenia & 1 & 3 & 5 & 1 & 58.8 & 35.3 \\
\hline Anemia & 1 & 3 & 2 & 0 & 35.3 & 11.8 \\
\hline Thrombocytopenia & 1 & 0 & 0 & 0 & 5.9 & 0.0 \\
\hline Nausea/vomiting & 4 & 1 & 3 & 0 & 47.1 & 17.6 \\
\hline Stomatitis & 0 & 1 & 0 & 0 & 5.9 & 0.0 \\
\hline Diarrhea & 1 & 2 & 1 & 0 & 23.5 & 5.9 \\
\hline Exanthema & 0 & 0 & 1 & 0 & 5.9 & 5.9 \\
\hline Alopecia & 3 & 3 & 0 & 0 & 35.3 & 0.0 \\
\hline Anorexia & 1 & 0 & 2 & 1 & 23.5 & 17.6 \\
\hline General malaise & 2 & 1 & 3 & 0 & 35.3 & 17.6 \\
\hline Abdominal pain & 0 & 1 & 0 & 0 & 5.9 & 0.0 \\
\hline Abdominal distension & 0 & 1 & 0 & 0 & 5.9 & 0.1 \\
\hline
\end{tabular}

B, After the first course.

\begin{tabular}{|c|c|c|c|c|c|c|}
\hline$n=6$ & G1 & $\mathrm{G} 2$ & G3 & G4 & All grades $(\%)$ & $>\mathrm{G} 3(\%)$ \\
\hline Leukocytopenia & 1 & 3 & 0 & 0 & 66.7 & 0.0 \\
\hline Neutropenia & 1 & 0 & 2 & 1 & 66.7 & 50.0 \\
\hline Anemia & 0 & 0 & 1 & 0 & 16.7 & 11.8 \\
\hline Nausea/vomiting & 3 & 0 & 0 & 0 & 50.0 & 17.6 \\
\hline Stomatitis & 1 & 0 & 0 & 0 & 16.7 & 0.0 \\
\hline Diarrhea & 0 & 2 & 1 & 0 & 50.0 & 5.9 \\
\hline Alopecia & 1 & 1 & 0 & 0 & 33.3 & 0.0 \\
\hline Anorexia & 2 & 1 & 1 & 0 & 66.6 & 17.6 \\
\hline General malaise & 1 & 1 & 0 & 0 & 33.3 & 17.6 \\
\hline Skin pigmentation & 1 & 0 & 0 & 0 & 16.7 & 0.1 \\
\hline
\end{tabular}

The rationale of this study was that a preceding administration of CPT-11 against S-1-resistant tumors may affect the downregulation of TS, resulting in recovered sensitivity to S-1 (6). We chose the sequential, rather than concurrent, administration of CPT-11 and S-1, for two reasons. The first was that the downregulation of TS requires a certain period of time to take effect following CPT-11 administration. The second was that avoidance of concurrent administration of these drugs may alleviate adverse effects and achieve a longer continuity period of chemotherapy.

Practical uses of the S-1/CPT-11 combination have been reported (7) as a second-line therapy. It was reported that the S-1 monotherapy, following failure of preceding S-1-containing regimens, was worthy of testing in larger-scale clinical trials. However, a recent study negatively evaluated this re-use of S-1 (8). This strongly suggests that continuation of S-1 administration following failure is pointless.

Consequently, the reversal of S-1 resistance indicates a potential way to reuse this drug. DPD, TS and CYP2A6 are involved in fluorouracil drug resistance, and modulators of these enzymes are candidates for concurrent or sequential usage. It is reported that inter-individual deviation in the gene expression and activity of these enzymes are associated with the ability to predict the effects of the chemotherapy (9-12). This suggests that tailor-made chemotherapy using intratumoral TS activity is possible. Using a xenograft model Fukushima et al reported that CPT-11 reversed chemo-resistance against 5-FU (6). The concept of our study originated from this application to clinical use.

As a result, the overall RR was $11.8 \%$ and the TTF, PFS and MST were 69, 71 and 381 days, respectively. The TTF and PFS were relatively short but the MST was long. However, with regard to our main aim of testing, the reversal of $\mathrm{S}-1$ resistance by a preceding treatment of CPT-11 was unfeasible since the RR following the first cycle was lower than estimated.

We investigated whether the time point of S-1 resistance acquisition affected the RR of this study by additional subset analyses. The previously resistant group tended to demonstrate a poor response, although the difference was not significant. This may be due to the small sample size.

Three patients withdrew from the study due to adverse events, rather than PD. A number of $\geq \mathrm{G} 3$ non-hematological adverse events were observed including 3 cases of nausea and vomiting, 1 of diarrhea, 1 of exanthema, 2 of anorexia, 3 of 
general malaise, and one $\geq \mathrm{G} 4$ hematological adverse event in the first course. Nine G3 hematological adverse events also occurred. Intensity of CPT-11 administration was found to be over the recommended dose, although we did not determine the uridine-diphosphate glucuronosyltransferase (UGT) 1A1 polymorphism (13).

A randomized, multicenter phase II/III study, JACCRO GC-05, comparing CPT-11/S-1 and CPT-11 monotherapy as a second-line therapy in S-1-resistant patients with advanced gastric cancer is currently under way. Future findings may thus reveal whether CPT-11 provides any favorable effects on S-1 resistance, which was not shown in the present study.

In conclusion, S-1/CPT-11 sequential therapy based on the reversal of S-1 drug resistance by CPT-11 was tested, although the interim analysis revealed a lower response rate than expected. This protocol was concluded as being unfeasible.

\section{References}

1. Murad AM, Santiago FF, Petroianu A, Rocha PR, Rodrigues MA and Rausch M: Modified therapy with 5-fluorouracil, doxorubicin and methotrexate in advanced gastric cancer. Cancer 72 : 37-41, 1993.

2. Glimelius B, Hoffman K, Haglund U, Nyrén O and Sjödén PO: Initial or delayed chemotherapy with best supportive care in advanced gastric cancer. Ann Oncol 5: 189-190, 1994.

3. Pyrhönen S, Kuitunen T, Nyandoto P and Kouri M: Randomized comparison of 5-fluorouracil, epirubicin, and methotrexate (FEMTX) plus supportive care with supportive care alone in patients with non-resectable gastric cancer. Brit J Cancer 71: 587-591, 1995.
4. Futatsuki K, Wakui A, Nakao I, et al: Late phase II study of irinotecan hydrochloride (CPT-11) in advanced gastric cancer. CPT-11 Gastrointestinal Cancer Study Group. Gan To Kagaku Ryoho 21: 1033-1038, 1994.

5. Koizumi W, Narahara H, Hara T, et al: S-1 plus cisplatin versus S-1 alone for first-line treatment of advanced gastric cancer (SPIRITS trial): a phase III trial. Lancet Oncol 9: 215-221, 2008.

6. Fukushima M, Sakamoto K, Ohshimo H, Nakagawa F and Taguchi T: Irinotecan overcomes the resistance to 5-fluorouracil in human colon cancer xenografts by down-regulation of intratumoral thymidylate synthase. Oncol Rep 24: 835-842, 2010.

7. Takiuchi H: Combination therapy with $\mathrm{S}-1$ and irinotecan (CPT-11) for advanced or recurrent gastric cancer. Gastric Cancer 12: 55-59, 2009.

8. Ono A, Boku N, Onozawa Y, et al: Activity of S-1 in advanced or recurrent gastric cancer patients after failure of prior chemotherapy, including irinotecan + cisplatin or fluorouracil (except S-1). Jpn J Clin Oncol 39: 332-335, 2009.

9. Miyamoto S, Ochiai A, Boku N, et al: Discrepancies between the gene expression, protein expression, and enzymatic activity of thymidylate synthase and dihydropyrimidine dehydrogenase in human gastrointestinal cancers and adjacent normal mucosa. Int J Oncol 18: 705-713, 2001.

10. Fukushima M, Morita M, Ikeda K and Nagayama S: Population study of expression of thymidylate synthase and dihydropyrimidine dehydrogenase in patients with solid tumors. Int J Mol Med 12: 839-844, 2003.

11. Ichikawa W, Takahashi T, Suto K, et al: Thymidylate synthase predictive power is overcome by irinotecan combination therapy with S-1 for gastric cancer. Br J Cancer 91: 1245-1250, 2004.

12. Takiuchi H, Kawabe S, Gotoh M and Katsu K: Thymidylate synthase gene expression in primary tumors predicts activity of S-1-based chemotherapy for advanced gastric cancer. Gastrointest Cancer Res 1: 171-176, 2007.

13. Innocenti F, Undevia SD, Iyer L, et al: Genetic variants in the UDP-glucuronosyltransferase 1A1 gene predict the risk of severe neutropenia of irinotecan. J Clin Oncol 22: 1382-1388, 2004. 